\title{
A Study of Knowledge Dimension and Cognitive Process Pattern of Cognitive Style Differences in STEM Cooperative Learning Environment
}

\author{
Peng-Chun Lin, Hsin-Ke Lu, and Yin-Chen Lin
}

\begin{abstract}
The purpose of this study was to investigate the differences of knowledge dimensions and cognitive process patterns of students with different cognitive styles in STEM collaborative learning activities. These students used Packet Tracer and Facebook to conduct collaborative problem-solving activities on network troubleshooting in order to learn related knowledge and skills; the researcher then utilized a hidden figure test to divide them into "Field-Independent Group (FI Group)" and "Field-Dependent Group (FD Group)" to investigate their learning outcomes. The results showed that their learning outcomes had improved.

The researcher further used "Revised Bloom's Taxonomy (RBT)" to code their group discussion contents and also content analysis and sequential analysis to investigate their knowledge dimensions and cognitive process patterns. The results indicated that knowledge dimensions of "FI Group" and "FD Group" were diverse; moreover, "FD Group" had an additional high-level knowledge dimension - C5 (Evaluate). For cognitive process patterns, students of "FD Group" had diverse transfers of knowledge deepening and cognitive process patterns; this meant that they could apply, analyze and even reflect known knowledge through the learning process. The participants of this study were the students studying introduction to IT at a university in Taiwan. In the future, it was expected that participant range could be enlarged so that more holistic references could be provided for teachers and researchers.
\end{abstract}

Index Terms-Cognitive style, knowledge dimension, cognitive process, collaborative problem solving, CPS.

\section{INTRODUCTION}

Besides developing students' professional skills, the purpose of university education also included developing their future competitiveness which included hard power and soft power. Hard power contained professional skills, technical application abilities, and foreign languages...etc.; soft power involved teamwork abilities, problem solving abilities and communication abilities. Therefore, we knew that higher education not only included teaching professional skills but also focused on interdisciplinary learning and developing their abilities of problem solving and collaborative learning in order to stimulate their creativity, imagination, and potential.

With the rapid development of information technology, STEM (Science, Technology, Engineering, Math) education has become a trend affecting global educational systems and

Manuscript received January 22, 2018; revised May 18, 2018.

The authors are with the Chinese Culture University, 231 Taipei, Taiwan (R.O.C.) (e-mail: pclin@sce.pccu.edu.tw, sklu@sce.pccu.edu.tw, yinclin@sce.pccu.edu.tw). it highly focused on the development of knowledge and skills - the necessary hard power in future workplace. Many scholars had investigated on using simulation-based tools to assist students to learn procedural knowledge and operation skills, and the results showed that their learning outcomes had improved [1], [2]. On the other hand, abilities of collaboration and problem solving were significant development in STEM; these were soft power. Plenty of scholars also investigated the teaching outcomes of using CPS (Collaborative Problem Solving) brought up by Nelson (1999) in STEM, and the results also indicated that using this strategy could improve students' learning outcomes and collaborative abilities [2]-[4].

Nevertheless, individual difference has always been a significant issue in education field, also an important index for adaptive teaching. In general studies, personal characteristics such as gender, age and education level were mostly used as individual difference; in educational studies, personal cognitive differences such as learning style, teaching style or perceived teaching style were focused (Lu \& Lin, 2012; Lin, Lu \& Fan, 2014). Several scholars also discovered that different cognitive styles would affect their learning behaviors in their studies [5]-[8].

The participants were 33 students taking the course "Intro. to Computer Networks" at one university in Taiwan. In the study, the researcher first adopted Wu's "Hidden Figure Test" [9] who revised Messick's (1962) hidden figure test based on Witkin's (1962) field-independence theory related to cognitive style and divided the students into "Field-Independent Group (FI Group) who were more independent and "Field-Dependent Group (FD Group)" who were more collaborative [5], [6], and then applied Collaborative Problem Solving (CPS) teaching strategies to investigate the differences of the learning outcome, knowledge dimension, and cognitive process pattern of these two groups with different cognitive styles in STEM field.

For analyzing learning outcome and learning behavior, the researcher used pre-test and post-test to investigate the differences. Besides, the researcher utilized Quantitative Content Analysis (QCA) and Lag sequential analysis (LSA) to investigate their differences of knowledge dimension and cognitive process pattern in CPS activities. The questions of this study included:

1) Are learning outcomes of learners of "FI Group" and "FD Group" different?

2) Are knowledge dimensions of learners of "FI Group" and "FD Group" different?

3) Are cognitive process patterns of learners of "FI Group" 
and "FD Group" different?

\section{RESEARCH METHOD}

\section{A. Research Tools}

- Hidden Figure Test

Cognitive difference was a significant issue in teaching field. Many researchers had different categories about cognitive styles. For instance, Riessman (1964) categorized cognitive styles into visual, auditory and kinesthetic according to sensory modalities [10]; Gregorc (1977) categorized them into concrete sequential, abstract random, abstract sequential and concrete random based on thinking patterns [11]. Witkin (1964) brought up field-independent (FI) and field-dependent (FD) based on the theory of psychological differentiation; it was widely applied in academic studies [5], [8], [9].

The researcher adopted Wu's "Hidden Figure Test" who revised Messick's (1962) hidden figure test to be the tool to measure cognitive styles [9]. Learners needed to find out one basic figure contained in the complicated geometric figures. Totally there were 32 complicated geometric figures; an example was shown in the following. A perfect score was 32. The scoring method was subtracting one fourth of wrong answer number from right answer number in order to calibrate the effect of guessing. Higher scores meant higher tendency toward field independence and vice versa.

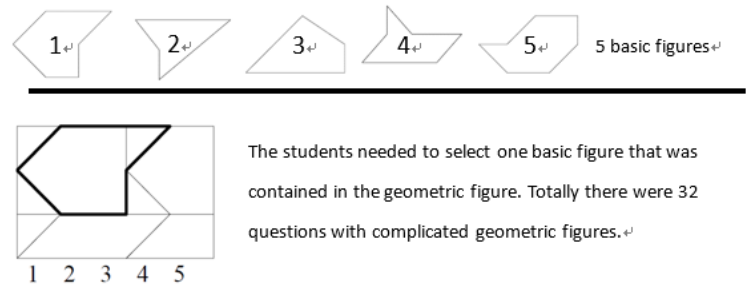

Fig. 1. Hidden figure test example.

- Network Troubleshooting Examination paper

The teaching activities of the collaborative problem solving were conducted in a course of Introduction to Information Technology at one university in Taiwan in this study, and therefore the researcher used the network troubleshooting examination paper designed by its teacher to perform the pre-test and post-test of network troubleshooting concept in order to investigate the difference of learning outcome before and after participating the activities. There were 8 single choice questions and one essay question, and the maximum score was 100 .

- Packet Tracer (PT)

In STEM educational field, the development of knowledge and skill played a crucial part and they were the necessary hard power needed in future workplace. For the collaborative problem-solving activities in this study, the researcher used Packet Tracer (PT) as the tool to train their operation skills. Packet Tracer is a software designed for learning network concepts and network equipment operation; its graphic interface with immediate feedbacks allowed students to design and simulate real-world network transmission to learn abstract knowledge hard to be observed. Frezzo, Behrens and Mislevy (2010) also mentioned that using Packet Tracer could provide students with a learning environment with better a system logic. Besides, other researchers also stated that using Packet Tracer in teaching could promote students' learning interest and learning outcome [12]-[14].

- Facebook (FB)

Many researchers had investigated the application of social network services (SNSs) in knowledge construction and cognitive process pattern in the teaching environment of collaborative learning [15], [16]. Facebook is one of the hottest SNSs, and people can easily share various multimedia information (including text and image) and do synchronous and asynchronous discussion through Facebook. It is widely used by college and university students, and therefore many researchers had investigated students' feelings about learning by using Facebook and their learning outcomes when it was used in official learning environments [17]-[19]. In this study, the researcher also utilized Facebook as the discussion platform in the teaching of collaborative problem solving so that these learners could use their habitual social network environment to discuss in the learning activities.

\section{B. Content and Behavioral Analysis}

In this study, the researcher used quantitative content analysis (QCA) and lag sequential analysis (LSA) to investigate knowledge dimensions of learners with different cognitive styles and analyze the cognitive process patterns of their discussion behaviors expecting to assist teachers and researchers to plan future teaching activities.

- Quantitative Content Analysis (QCA)

The researcher used the cognitive process dimension of Revised Bloom's Taxonomy (RBT) edited by Anderson and Krathwohl (2001) to code learners' discussion data in FB and conduct the quantitative content analysis (QCA) in order to investigate the coding level of their knowledge dimension.

TABLE I: THE COGNITIVE PROCESS CODING SCHEME OF RBT (ANDERSON \& KRATHWOHL, 2001)

\begin{tabular}{|c|c|c|}
\hline Code & Phase/content & Description \\
\hline $\mathrm{C} 1$ & Remember & $\begin{array}{l}\text { To access relevant knowledge form long-term } \\
\text { memory. }\end{array}$ \\
\hline $\mathrm{C} 2$ & Understand & $\begin{array}{l}\text { To make sense of acquired knowledge; to associate } \\
\text { new knowledge with past experiences. }\end{array}$ \\
\hline $\mathrm{C} 3$ & Apply & $\begin{array}{l}\text { To do a job or solve a problem through application } \\
\text { (procedures). }\end{array}$ \\
\hline $\mathrm{C} 4$ & Analyze & $\begin{array}{l}\text { To break down and analyze each component of } \\
\text { knowledge and note the relationship between the part } \\
\text { and the whole. }\end{array}$ \\
\hline $\mathrm{C} 5$ & Evaluate & To judge and evaluate based on criteria and standards. \\
\hline C6 & Create & $\begin{array}{l}\text { To piece different elements together and form a } \\
\text { complete and functional whole. To form a new } \\
\text { structure by re-assembling elements through the } \\
\text { mental process. }\end{array}$ \\
\hline $\mathrm{C} 7$ & $\begin{array}{l}\text { Irrelevant to the } \\
\text { project topic }\end{array}$ & Other types of knowledge irrelevant to project topic. \\
\hline
\end{tabular}

- Lag sequential analysis (LSA)

Lag sequential analysis (LSA) was brought up by Bakeman \& Gottman (1997) in the book "Observing interaction: An introduction to sequential analysis" [20]; its major concept was that if data obtained by observation was sequential, then it could be coded and relations among these codes could be investigated, and behaviors with significant correlation (z-score) could be found. This method currently was used in observing learners' process patterns during learning activities by many scholars [15], [21]. In this study, GSEQ (Generalized Sequential Querier) was used to conduct 
a sequential analysis of cognitive process.

\section{Experimental Design}

- Participants

The participants of this study were 33 students taking the course "Intro. to Computer Networks" at one university in Taiwan; this course was elective, and hence they came from sophomores, juniors and seniors of "Department of Information Management", "Department of Digital Media Design" and "Department of Business Management".

- Experimental Process

In this study, the researcher conducted the pre-test of Hidden Figure Test and Network Troubleshooting at the 16th week of the semester, and the researcher divided the students into several teams (4-5 people in a team) according to their pre-test scores and made sure that there was so significant difference among the pre-test scores of each team. Then, the researcher categorized these teams into "FI Group" and "FD Group" according to the results of hidden figure test.

The collaborative problem-solving activity was conducted at the $17^{\text {th }}$ week of the semester. All students needed to use Packet Tracker and Facebook to solve the network troubleshooting tasks assigned by the instructor, and then finish the post-test of network troubleshooting ability after the activity so that their abilities could be understood.

Finally, the researcher collected all groups' discussion contents on Facebook and then conducted the quantitative content analysis (QCA) on them according to Revised Bloom's Taxonomy (RBT) to investigate knowledge dimension distribution of different groups. Later, the researcher used lag sequential analysis (LSA) to compare cognitive process patterns of different groups.

\section{RESULT AND DISCUSSION}

\section{A. Analysis on Learning Outcome}

The researcher first analyzed the pre-test and post-test on the network troubleshooting ability of these 33 learners; the results showed that their post-test score (69.697) was better than that of pre-test (50.788). $T=-4.329$ and $p=.000<.001$ (as Table 2 showed) indicated that their score had improved after participating in the collaborative problem-solving activity based on Facebook and Packet Tracker designed by the researcher. The results corresponded to those of many researchers; the high popularity and high acceptance of Facebook among college and university students with the highly precise and graphic simulated environment of packet tracer would advance students' learning outcomes in network concept and troubleshooting [15], [16], [22].

TABLE II: ANALYSIS OF ALl STUDENTS' PRE-TEST AND POST-TEST BEFORE AND AFTER PARTICIPATING THE TEACHING ACTIVITY

\begin{tabular}{lccccc}
\hline \hline \multicolumn{1}{c}{ Test } & Number & Mean & St. Deviation & $t$ & $p$ \\
\hline Pre-Test & 33 & 50.788 & 20.031 & & \\
Post-Test & 33 & 69.697 & 19.449 & -429 & $p=.000<.01$ \\
\hline \hline
\end{tabular}

The researcher divided the students into 8 teams (4-5 people in each team) according to their pre-test scores of Network Troubleshooting done at the 16th week. Among these 8 teams, the researcher then categorized 4 teams as "FI
Group" and the other 4 teams as "FD Group" according to their results of the hidden figure test. The mean of hidden figure test of 16 students of "FI Group" was 10.44; on the other hand, the mean of hidden figure test of 17 students of "FD Group" was 1.96 .

The researcher then conducted an independent sample analysis of covariance (ANCOVA). In slope homogeneity test, $\mathrm{F}=1.371$ and $p=0.251>0.05$; this meant that the pre-test scores of "FI Group" and "FD Group" were not significantly different. The result of covariance analysis $(\mathrm{F}=3.254$ and $p=0.081>0.05$ ) showed that their post-test scores also were not significantly different.

The reasons might include that the students had limited time to join in the learning activity, their task objectives were clear, and they were familiar with the tools of Packet Tracer and Facebook, all students had completed their learning and joined in team discussion enthusiastically, and therefore the post-test scores of "FI Group" and "FD Group" were not significant different.

\section{B. Analysis of Knowledge Dimension}

The researcher had coded all teams' discussion contents on Facebook according to Revised Bloom's Taxonomy (RBT) and conducted the quantitative content analysis (QCA). To ensure the high credibility of coding, two coders did the coding so that scorer reliability could be ensured in this study. Cohen's kappa value reached to $K=.777>.750$ of the two coders; this meant that they had excellent agreement.

There were totally 210 items in "FI Group" discussion and 193 items in "FD Group" discussion; their knowledge dimensions were compared in the following figure.
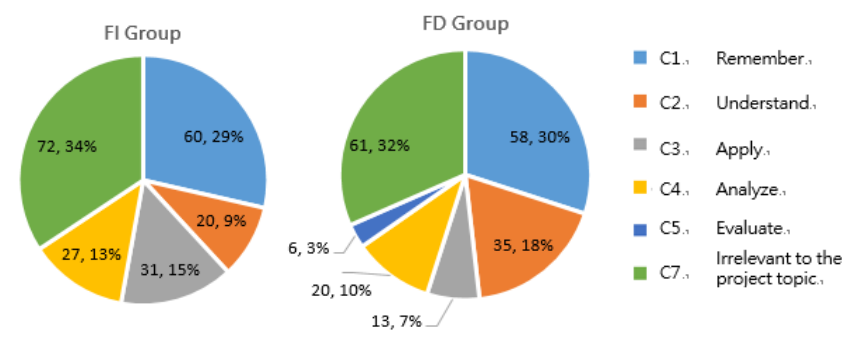

Fig. 2. Comparison of the coding of "FI Group" and "FD Group".

Fig. 2 showed that discussion items were not significantly different in number; knowledge dimensions of "FI Group" included 5 dimensions- C1 (Remember), C2(Understand), C3(Apply), C4(Analyze) and C7(Irrelevant to the project topic), and $\mathrm{C} 7$ accounted for the highest percentage (irrelevant to the project topic) which was $34 \%$. The next was $30 \%$ - C1 (Remember); this meant that the students could recall the knowledge that the teacher taught. C3 (Apply) accounted for $15 \%$ and C4 (Analyze) accounted for $13 \%$; this indicated that the students could apply their knowledge to the operating environment and further could analyze the circumstances in the environment.

For "FD Group", the highest one was C7 (32\%), and the following ones were $\mathrm{C} 1$ (58\%), C2 (18\%), C4 (10\%), C3 (7\%) and C5 (3\%). Different from "FI Group", it had C5 (Evaluate); this meant that students with higher dependence could stimulate contents with a higher level of knowledge in the collaborative tasks and this was appropriate in instructional design. In both groups, the percentage of C7 
was the highest; this result was not necessarily negative to discussion. Many studies indicated that discussions irrelevant to the project topic might improve teams' coherence [16], [23]. This corresponded to the results of this study; many items of C7 included asking whether classmates needed helps or making sure that all team members had agreement.

\section{Sequential Analysis}

The researcher further conducted the lag sequential analysis (LSA) on QCA results in order to investigate two teams' differences in cognitive process patterns. FI Group's z-score was shown in Table III and FD Group's z-score was shown in Table IV.

TABLE III: Z-SCORE OF FI GROUP

\begin{tabular}{crrrrr}
\multicolumn{7}{c}{ TABLE III: Z-SCORE OF FI GROUP } \\
\hline \hline Code & C1 & C2 & C3 & C4 & C7 \\
\hline C1 & 0.66 & -1.53 & 0.65 & 0.93 & -1.21 \\
C2 & -1.73 & 1.22 & $\mathbf{1 . 9 8}$ & -0.79 & -0.37 \\
C3 & -0.2 & 1.03 & -0.01 & 0.73 & -1.45 \\
C4 & 1.73 & -1.1 & -0.71 & 0.97 & -1.36 \\
C7 & -0.65 & 0.96 & -1.74 & -1.85 & 3.93 \\
\hline \hline
\end{tabular}

\begin{tabular}{crrrrrr}
\multicolumn{7}{c}{ TABLE IV: Z-SCORE OF FD GROUP } \\
\hline \hline Code & C1 & C2 & C3 & C4 & C5 & C7 \\
\hline C1 & -0.78 & 0.65 & 0.11 & $\mathbf{2 . 5 1}$ & 1.16 & -1.70 \\
C2 & 0.65 & 1.31 & -1.30 & -1.12 & -1.01 & 0.06 \\
C3 & -0.97 & -0.35 & $\mathbf{2 . 0 5}$ & -0.7 & 1.15 & -0.12 \\
C4 & 1.31 & 0.36 & 1.02 & -0.99 & -0.90 & -1.28 \\
C5 & $\mathbf{1 . 9 7}$ & 0.29 & -0.61 & -0.52 & -0.47 & -1.36 \\
C7 & -1.06 & -2.09 & -0.76 & -0.41 & -0.19 & $\mathbf{3 . 6 0}$ \\
\hline \hline
\end{tabular}

The researcher had compared these two groups' behavioral sequential analysis as Fig. 3 showed. The results showed that the students of "FI Group" seldom had the transfer and deepening of knowledge dimension in the task of collaborative problem solving, and they only had the transfer from C2 (Understand) to C3 (Apply) and the self-transfer of C7 (Irrelevant to the project topic). This represented that these students could deepen their knowledge to actual application during the activity; this was positive in learning. On the other hand, students of "FD Group" had more processes of knowledge transfer and deepening. The transfer of $\mathrm{C} 1$ (Remember) to $\mathrm{C} 4$ (Analyze) showed that they could recall what the teacher taught and transfer them to analyzing the operating environment; this was the phenomenon of lower level knowledge dimension transferring to higher level knowledge dimension. This indicated that they could totally digest what the teacher taught. The transfer of $\mathrm{C} 2$ (Understand) to C3 (Apply) was the same with the other team; this represented a positive feedback of learning. The special thing was that it had the transfer of C5 (Evaluate) to C1 (Remember); this indicated that they could remember what the teacher taught from "evaluate" knowledge dimension. This was highly valuable because it meant that they could reflect and respond to the existing knowledge; this was highly positive.

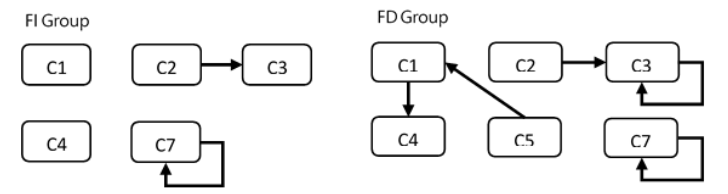

Fig. 3. The behavioral sequential analysis of FI group and FD group.

\section{CONCLUSION}

The purpose of this study was to investigate the differences of knowledge dimension and cognitive process pattern of students with different cognitive styles in a STEM collaborative learning environment. The learners utilized Packet Tracer and Facebook to conduct the collaborative problem-solving activities on network troubleshooting in order to learn related knowledge and skills. The researcher then used a hidden figure test to categorize them into "FI Group" and "FD Group" in order to investigate the differences of learning outcome, knowledge dimension and cognitive process pattern of discussion. The results indicated that their learning outcomes had improved; this had corresponded to the results of other researches [15], [16], [22], [24]. Nevertheless, the learning outcomes of "FI Group" and "FD Group" were not significantly different.

The researcher further analyzed their discussion contents on Facebook and used Revised Bloom's Taxonomy (RBT) to code them and analyze the knowledge dimension of discussion contents and investigate their cognitive process patterns. The results showed that the knowledge dimensions of "FI Group" and "FD Group" were both diverse including C1 (Remember), C2 (Understand), C3 (Apply), C4 (Analyze) and $\mathrm{C} 7$ (Irrelevant to the project topic). Moreover, "FD Group" had a high-level knowledge dimension - C5 (Evaluate). For cognitive process pattern, the learners of "FD Group" had multiple transfers of knowledge deepening and process pattern during the collaborative problem-solving activity. This showed that they could apply and analyze the known knowledge through the learning process $(\mathrm{C} 1 \rightarrow \mathrm{C} 4$, C2 -> C3) and even could reflect (C5 -> C1); this was highly positive feedbacks for the learners. This also showed that this type of learning activity could promote "field-dependent" learners' discussions and their learning outcomes. The participants of this study were the students taking the course of "Introduction to IT" at one university in Taiwan; it was expected that more diverse participants can be included in the future studies to provide teachers and researchers with more holistic references.

\section{REFERENCES}

[1] O. Noroozi, H. J. A. Biemans, M. C. Busstra, M. Mulder, V. Popov, and M. Chizari, "Effects of the Drewlite CSCL platform on students' learning outcomes," Strategies and Applications, 2012, pp. 276-289.

[2] P. C. Lin, H. T. Hou, S. Y. Wu, and K. E. Chang, "Exploring college students' cognitive processing patterns during a collaborative problem-solving teaching activity integrating Facebook discussion and simulation tools," Internet and Higher Education, vol. 22, pp. 51-56, 2014.

[3] C. M. Achilles and S. P. Hoover, "Transforming administrative praxis: The potential of problem-based learning (PBL) as a school improvement vehicles for middle and high schools," Annual Meeting of the American Educational Research Association, New York, 1996.

[4] L. M. Nelson, "Collaborative problem solving," Instructional-Design Theoriesand Models: A New Paradigm of Instructional Theory, Mahwah, N.J: Erlbaum Associates, 1999.

[5] H. A. Witkin, C. A. Moore, D. R. Goodenough, and P. W. Cox, "Field dependent and field independent cognitive styles and their educational implication," Review of Educational Research, vol. 47, no. 1, pp. 1-64, 1962.

[6] S. Messick, "Individuality in learning: Implications of cognitive styles and creativity for human development," San Francisco: Jossey-Bass Park, CC, 1962.

[7] C. H. M. Lee, Y. W. Cheng, S. Rai, and A. Depickere, "What affect student cognitive style in the development of hypermedia learning system?" Computers \& Education, vol. 45, pp. 1-9, 2005. 
[8] H. K. Lu and P. C. Lin, "A study on the effect of cognitive style in the field of STEM on collaborative learning outcome," International Journal of Information and Education Technology, vol. 8, no. 3, pp. 194-198, 2017.

[9] J. J. Wu, "Hidden figure test," Yuan-Liou, Taipei, 1974.

[10] F. Riessman, "Styles of learning," Psychology fo Education, New York, 1964.

[11] A. Gregorc, "A new definition for Individurus," NASSP BuUetin, pp. 20-26, 1977

[12] D. C. Frezzo, J. T.Behrens, and R. J. Mislevy, "Design patterns for learning and assessment: Facilitating the introduction of a complex simulation-based learning environment into a community of instructors," Journal of Science Education and Technology, vol. 19, no. 2, pp. 105-114, 2010.

[13] C. Goldstein, S. Leisten, K. Stark, and A. Tickle, "Using a network simulation tool to engage students in active learning enhances their understanding of complex data communications concepts," in Proc. the Seventh Australasian Computing Education Conference (ACE2005), Newcastle: Australia, 2005.

[14] H. K. Lu and P. C. Lin, "A study of the impact of collaborative problem-solving strategies on students' performance of simulation-based learning- A case of network basic concepts course," International Journal of Information and Education Technology, vol. 7 , no. 5, pp. 361-366, 2016.

[15] P. C. Lin, H. T. Hou, S. M. Wang, and K. E. Chang, "Analyzing knowledge dimensions and cognitive process of a project-based online discussion instructional activity using Facebook in an adult and continuing education course," Computers and Education, vol. 60, no. 1 , pp. 110-121, 2013.

[16] P. C. Lin, H. T. Hou, S. M. Wang, and K. E. Chang, "Analyzing knowledge dimensions and cognitive process of a project-based online discussion instructional activity using Facebook in an adult and continuing education course," Computers and Education, 2014.

[17] R. Junco, "The Relationship between frequency of facebook use, participation in Facebook activities, and student engagement," Computers \& Education, vol. 58, no. 1, pp. 162-171, 2012.

[18] M. K. Kabilan, , N. Ahmad, and M. J. Z. Abidin, "Facebook: An online environment for learning of English in institutions of higher education?" Internet and Higher Education, vol. 13, no. 4, pp. 179-187, 2010.

[19] P. A. Kirschner and A. C. Karpinski, "Facebook and academic performance," Computers in Human Behavior, vol. 26, no. 6, 1237-1245, 2010.

[20] R. Bakeman and J. M. Gottman, "Observing interaction: An introduction to sequential analysis," New York: Cambridge University Press, 1997.
[21] P. C. Lin, H. K. Lu, and S. M. Fan, "Exploring the effect of perceived teaching style on behavioral intention toward the moodle reading system," International Journal: Emerging Technologies and Learning, vol. 9, no. 3, pp. 64-67, 2014.

[22] S. Y. Wu, H. T. Hou, and W. Y. Hwang, "Exploring students' cognitive dimensions and behavioral patterns during a synchronous peer assessment discussion activity using Instant Messaging," The Asia-Pacific Education Researcher, vol. 21, no. 3, pp. 442-453, 2012.

[23] H. T. Hou and S. Y. Wu, "Analyzing the social knowledge construction behavioral patterns of an online synchronous collaborative discussion instructional activity using an instant messaging tool: A case study," Computers \& Education, vol. 57, no. 2, pp. 1459-1468, 2011.

[24] H. K. Lu and P. C. Lin, "Effects of interactivity on students' intention to use simulation-based learning tool in computer networking education," in Proc. 3rd International Conference on Next Generation Information Technology, 2012.

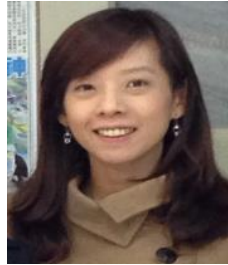

Peng-Chun Lin is an assistant professor of the Department of Information Management in Chinese Culture University. She is also the chief officer of the International Information Certification Cooperation Center at Chinese Culture University in Taiwan. Her academic interests focus on e-learning, network community development, application of simulation learning and recently on optimal network management and corporate structure.

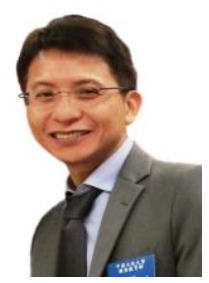

Hsin-Ke Lu is the director of information management (master programs for working learners) and the CEO of School of Continuing Education at Chinese Culture University in Taiwan. $\mathrm{He}$ is the chairman of the Association of Continuing Education of Colleges and Universities in Taiwan and the Chairman of Cisco Networking Academy. His academic interests focus on e-learning, corporate structure, lifelong learning, and information system planning (enterprise architecture).

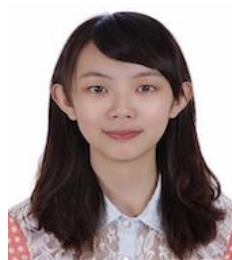

Yin-Chen Lin is an undergraduate student of the Department of Information Management, Chinese Culture University. Meanwhile she secured her job in $\mathrm{CCU}$ as a software engineer responsible for deploying of app design and app programming. 